\title{
Prendre le temps: pour toi, pour moi, pour nous
}

\section{Hans Kurt}

Dr méd., représentant de la FMH au comité central de la «Journée des malades»

La pression du temps n'est pas réservée aux seuls médecins, nous y sommes tous soumis. Il faut être rapide, efficace, le travail doit être optimisé selon un facteur prépondérant: le temps. C'est pourquoi la «Journée des malades» de cette année est consacrée à cette problématique importante.

La médecine s'assimile progressivement à un processus de production. "Plus on considère la médecine comme un processus de production, plus on valorise l'activisme, encourage les moyens d'action, pénalise l'écoute, calcule le temps d'intervention, ignore le temps de consultation, érige l'augmentation de la cadence en valeur phare et, enfin, considère la prudence et l'attention comme des freins au fonctionnement», relève le Prof. Giovanni Maio de Fribourg-enBrisgau dans un article de la revue «Synapse».

Les enquêtes sur les interactions médecin-patient montrent que les patients sont fréquemment interrompus après 10 à 20 secondes lorsqu'ils veulent énoncer les motifs de leur visite, avant même d'avoir le temps de décrire leur situation, de se sentir compris et que le médecin puisse finalement savoir de quoi il s'agit. Si les patients n'étaient pas interrompus, ils parleraient 90 secondes. Prendre le temps d'écouter ou justement économiser sur ce temps d'écoute sont des facteurs essentiels du quotidien des malades, de leurs proches, des médecins et du personnel infirmier. Dans le cadre de l'économisation de la santé, incluant la règle selon laquelle tous nos actes et leurs résultats doivent être convertis en chiffres, le facteur temps joue un rôle prépondérant: il doit donc être mesuré et relevé. Nous connaissons la saisie du temps, le tarif au temps, la durée d'intervention, la durée de séjour, la durée de processus. Aujourd'hui, le temps tourne de plus en plus vite en médecine.

\section{Moins de temps pour les activités avec les patients}

La durée moyenne d'hospitalisation en Suisse n'a cessé de baisser ces dernières années, mais les hôpitaux et les cliniques continuent malgré tout de remplir leurs lits. Différentes études, notamment au CHUV et à l'Hô- pital cantonal de Baden, montrent que les médecinsassistants passent chaque jour respectivement 100 et 90 minutes au chevet des patients, ce qui correspond à un contact direct avec les patients d'à peine 15 minutes et ce pour un temps de travail moyen de 11,5 heures par jour. En revanche, ils passent trois fois plus de temps devant un ordinateur. D’après une étude de la FMH, les activités auprès des patients en soins somatiques aigus et en psychiatrie ne correspondent plus qu'à un tiers environ du temps de travail. La charge administrative augmente également dans les cabinets ambulatoires et, depuis l'intervention tarifaire du Conseil fédéral, les prestations sont désormais saisies par tranche d'une minute.

Ces dernières années, le lien entre maladie et capacité de travail est devenu un sujet central de notre société, le travail étant une preuve de santé, de reconnaissance et de sens de la vie. Nous parlons beaucoup de réinsertion professionnelle et les milieux politiques se sont fixé comme objectifs de s'attaquer aux fraudes à l'assurance et de remettre les patients le plus rapidement possible au travail. Mais c'est oublier que beaucoup d'entre eux retournent travailler même s'ils sont

\section{La Journée des maldes}

La «Journée des malades» est une association d'utilité publique regroupant des organisations de patients, les ligues de la santé, des associations professionnelles, la Conférence suisse des directrices et directeurs cantonaux de la santé (CDS) et la FMH. Fondée en 1939, elle sensibilise chaque année la population à un thème particulier et entend ainsi favoriser les relations entre les malades et les bien-portants, contribuer à une meilleure compréhension des besoins des malades et rappeler aux personnes en bonne santé leurs devoirs envers les souffrants. Cette année, la "Journée des malades" aura lieu le 4 mars et sera l'occasion de plusieurs manifestations publiques.

Pour de plus amples informations: www.journeedesmalades.ch 
encore malades car ils ont le sens du devoir et peur de perdre leur emploi. Une enquête réalisée par Travail. Suisse relève que le présentéisme est très répandu en Suisse. Pas moins de $30 \%$ des personnes interrogées ont répondu travailler souvent, voire très souvent, même en étant malades. Vu sous cet angle, il est évident que le temps pour une guérison complète fait souvent défaut. L'utilisation problématique de médicaments, en particulier les analgésiques et certains psychotropes, nous laisse croire que nous économisons du temps alors qu'en réalité, de nouveaux problèmes sont créés, engendrant d'autres difficultés.

D'un côté, il y a le temps de la maladie. De l'autre, celui des proches aidants d'enfants ou de personnes âgées. Selon les chiffres de l'Office fédéral de la statistique, un tiers de la population suisse âgée de 15 ans et plus a accompli un bénévolat informel, sous forme d'aide de proximité, de garde d'enfants, de prestations et de soins à des parents ou à des connaissances. Ces tâches représentent chaque année près de 64 millions d'heures de soins et d'assistance, soit un équivalent de 3,5 milliards de francs. Cette forme de soutien va encore augmenter ces prochaines années avec le vieillissement de la population. Pour accomplir leurs tâches, les proches aidants ont également besoin de soutien et ils ont des attentes de la part des corps médical et infirmier. Lors d'une enquête auprès de proches aidants de personnes atteintes de troubles psychiques, $81 \%$ d'entre eux ont répondu que le plus important était de mettre davantage de temps à disposition pour les entretiens avec les proches. Ces derniers ont besoin de temps pour recevoir les informations, avoir des entretiens et ressentir que leur travail d'accompagnement est reconnu. Ils en ont également besoin pour leur propre repos et leurs propres aspirations.

Correspondance: Dr Hans Kurt Spécialiste en psychiatrie et psychothérapie Bielstrasse 109 CH-4500 Soleure kurt[at]solnet.ch

\section{Avoir du temps est capital pour tout rétablissement}

Avoir ou ne pas avoir de temps joue un rôle fondamental dans notre activité médicale et la médecine en

\section{Ursula Steiner-König}

Fin août 2017, la Dresse Ursula Steiner-König est décédée à Bâle à l'âge de 78 ans. Elle a siégé pendant plus de dix ans en tant que déléguée de la FMH au Comité central de la "Journée des malades». Par ses différents engagements politiques, notamment en tant que vice-présidente de la $\mathrm{FMH}$, elle a contribué avec énergie à la "Journée des malades" en partageant son immense expérience et ses compétences. Nous la gardons dans notre mémoire et la remercions vivement pour son engagement.

général. Différentes enquêtes montrent que les médecins qui consacrent suffisamment de temps aux entretiens avec les patients et les proches exercent leur profession avec plus de satisfaction. La relation entre le médecin et le patient revient au centre de la médecine; prendre le temps et donner de son temps constitue un facteur essentiel à la réussite d'un traitement. Prenez donc le temps pour vos patients, pour leurs proches mais aussi pour votre entourage, que ce soit au travail ou dans votre vie privée. Prenez le temps de prendre un café avec vos assistantes médicales, de discuter avec le personnel infirmier. Racontez-vous vos difficultés au quotidien mais aussi les rencontres positives et les patients gratifiants. Prenez aussi le temps de lever le pied lors de journées chargées, de vous asseoir et de regarder par la fenêtre pour savourer le temps qui passe. Lorsque vous rentrez à la maison après une journée exténuante, vous devriez peut-être vous asseoir à table avec votre famille au lieu de vous plonger directement dans une revue médicale spécialisée, et jouer avec elle aux cartes et boire calmement un thé avant de vous offrir ensuite le temps dont vous avez besoin pour votre repos et votre détente.

Le thème de la "Journée des malades» de cette année n'est pas seulement une question pour les malades mais aussi un sujet qui nous concerne tous et tout particulièrement nous médecins qui, si souvent, croyons ne pas avoir de temps. 